\title{
Combined intra-arterial and intravenous chemotherapy for unresectable, advanced gastric cancer has an improved curative effect compared with intravenous chemotherapy only
}

\author{
JINFENG WANG $^{1}$, HUACUN SHI ${ }^{1}$, GUANG YANG ${ }^{2}$, GUANGJIE HAN ${ }^{1}$, MAN ZHAO $^{1}$, XIAOLING DUAN $^{1}$, \\ LILI MI ${ }^{1}$, XIN HAN ${ }^{1}$, NING LI ${ }^{1}$, JIANFEI SHI ${ }^{1}$, XIAOLEI YIN ${ }^{1}$ and FEI YIN ${ }^{1}$ \\ Departments of ${ }^{1}$ Gastroenterology and ${ }^{2}$ Radiology, The Fourth Hospital of Hebei Medical University, \\ Shijiazhuang, Hebei 050035, P.R. China
}

Received April 25, 2017; Accepted November 29, 2017

DOI: $10.3892 / \mathrm{ol} .2018 .8068$

\begin{abstract}
The aim of the present study was to evaluate the efficacy and safety of a combination of intra-arterial and intravenous chemotherapy in the treatment of unresectable, advanced gastric cancer, and assess which patients are likely to benefit from combined treatment. The clinical data of 128 patients diagnosed with unresectable, advanced gastric cancer at The Fourth Hospital of Hebei Medical University (Shijiazhuang, China) from January 2009 to September 2012 were retrospectively analyzed. The patients were divided into two groups as follows: Those who received regional intra-arterial chemoembolization plus systemic chemotherapy (combined group; $n=62$ ) and those who received systemic chemotherapy only (venous group; $n=66$ ). The clinical response, overall survival (OS) and toxic effects in the two groups were compared. Univariate and multivariate analyses were performed to identify the primary factors affecting the survival time of patients in the combined group. The overall response rate was significantly increased $(35.5 \%)$ in the combined group compared with the venous group (19.7\%; $\mathrm{P}=0.045)$. The median OS was 14 months in the combined group and 13 months in the venous group, and the 1-year and 2-year survival rates in the two groups were 45.2 and $9.7 \%$, and 40.9 and $6.1 \%$, respectively. There were significant differences between the survival curves $(\mathrm{P}=0.044)$. The median time to progression in the combined group and the venous group was 10 months and 6 months, respectively, and the difference was statistically significant $(\mathrm{P}=0.003)$. Multivariate analysis revealed that tumor-node-metastasis (TNM)-stage and the degree of
\end{abstract}

Correspondence to: Professor Fei Yin, Department of Gastroenterology, The Fourth Hospital of Hebei Medical University, 169 Tianshan Street, Shijiazhuang, Hebei 050035, P.R. China

E-mail: yinfei_4y@sina.com

Key words: unresectable gastric cancer, intra-arterial chemotherapy, intravenous chemotherapy, combined treatment tumor staining were independent factors affecting OS. No differences in adverse reactions between the two groups were observed $(\mathrm{P}>0.05)$. The combination of intra-arterial and intravenous chemotherapy may effectively improve the rate of clinical response, prolong OS and time to symptomatic progression in patients with unresectable, advanced gastric cancer, in particular those with an earlier TNM stage and distinct tumor staining.

\section{Introduction}

Gastric cancer is one of most common carcinomas and the second leading cause of cancer-associated mortality globally (1). Furthermore, gastric cancer has a poor prognosis and the five-year survival rate is $<20 \%$ (2). The annual incidence of gastric cancer in China accounts for $>40 \%$ of the incidence worldwide (3). As the majority of patients are diagnosed at an advanced stage, numerous patients lose the opportunity for surgery. Previously, the majority of patients received systemic chemotherapy, which was characterized by a low concentration of the drug in the local lesion, little benefit, and numerous side effects. With the development of interventional technology, multiple national and international researchers (4-6) have demonstrated that intra-arterial chemoembolization through the tumor feeding arteries increases the drug concentration, improves the curative effect and reduces the number of adverse reactions. However, intra-arterial chemotherapy has little effect against circulating tumor cells, which are distributed throughout the body.

In order to solve the problems above, the present study investigated a novel therapeutic concept: A combination of intra-arterial and intravenous chemotherapy for unresectable, advanced gastric cancer, which considers local and systemic aspects to achieve a complementary effect. In the present study, our group retrospectively analyzed the clinical data of 128 patients with unresectable, advanced gastric cancer, in order to compare the short-term effects, long-term effects and adverse reactions in patients who received regional intra-arterial chemoembolization plus systemic chemotherapy (combination group) with those who received systemic chemotherapy only 
(venous group), and assessed which patients were suitable for combined treatment.

\section{Patients and methods}

Patients. A total of 128 patients who were diagnosed with unresectable, advanced gastric cancer at The Fourth Hospital of Hebei Medical University (Shijiazhuang, China) between January 2009 and September 2012 were enrolled in the present study. The present study was reviewed and approved by the Institutional Review Board of The Fourth Hospital of Hebei Medical University, and written informed consent was obtained from patients prior to treatment. The patients were divided into two groups: Those who received regional intra-arterial chemoembolization plus systemic chemotherapy (combined group; $n=62$ ) and those who received systemic chemotherapy only (venous group; $\mathrm{n}=66$ ). There were 50 males and 12 females in the combined group and the average age was $63.0 \pm 11.2$ years (range, $30-88$ years). There were 44 cases of cardiac cancer, 9 cases of gastric fundus carcinoma and 9 cases of gastric antrum carcinoma in the combined group. Twenty-two patients had poorly differentiated carcinoma and 40 patients had moderately well-differentiated carcinoma. The tumor-node-metastasis (TNM) stage distribution (7) was as follows: 26 IIIC-stage cases and 36 IV-stage cases. There were 7 patients without lymph node metastasis, 32 patients with local lymph node metastasis and 23 patients with distant lymph node metastasis. A total of 19 patients had hepatic metastasis and 21 patients had organ metastases. There were 49 males and 17 females in the venous group and the average age was $60.4 \pm 12.5$ years (range, $31-90$ years). There were 38 cases of cardiac cancer, 14 cases of gastric fundus carcinoma and 14 cases of gastric antrum carcinoma. A total of 20 patients had poorly differentiated carcinoma, and 46 patients had moderately well-differentiated carcinoma. The TNM stage distribution was as follows: 20 IIIC-stage cases and 46 IV-stage cases. There were 4 patients without lymph node metastasis, 41 patients with local lymph node metastasis and 21 patients with distant lymph node metastasis. A total of 21 patients had hepatic metastasis and 31 patients had organ metastases.

Equipment and materials. Digital subtraction angiography was performed with a GE4100 Innova machine (GE Healthcare, Chicago, IL, USA). RH, 5F-Yashiro and Corba catheters, Progreat and Stride microcatheters, and the micro godet system were from Terumo Corporation (Tokyo, Japan). Gelatin sponge particles (150-350, 560-710 and 710-1,000 $\mu \mathrm{m})$ were from Hangzhou Alicon Pharm Sci \& Tec Co., Ltd. (Hangzhou, China).

Inclusion criteria. All the patients in the present study had a histologically confirmed gastric carcinoma. Surgery is the first choice of treatment for patients with operable gastric cancer, and it has a substantial influence on prognosis. Considering this, operable patients with II-IIIB stage tumors and the patients who underwent surgical treatment were removed from the research, and the included patients had unresectable, IIIC-IV advanced stage gastric cancer. Patients diagnosed with unresectable, advanced gastric cancer should meet the following criteria: i) Diagnosis of stage IIIC or IV metastasis of lymph nodes by enhanced computed tomography (CT) or magnetic resonance imaging (MRI); ii) tumor infiltration and encompassment of major blood vessels, including the hepatic artery, celiac artery and portal vein; iii) distant metastasis (for example, liver metastasis). To be included, patients needed an Eastern Cooperative Oncology Group performance status (8) of $\leq 2$. The patients also needed to have adequate bone marrow function (neutrophilic granulocyte count $\geq 1.5 \times 10^{9} / 1$ and platelet count $\left.\geq 100 \times 10^{9} / 1\right)$, and adequate blood coagulation, renal and hepatic function to be included.

Exclusion criteria. Patients who had completed previous anti-cancer therapies prior to inclusion, including adjuvant chemotherapy, radiation therapy, surgery or biotherapy were excluded, as were patients with resectable II-IIIB TNM stage gastric cancer. Other exclusion criteria were as follows: Being pregnant or currently breast-feeding, the presence of other malignant tumors, massive ascites, poor blood coagulation, or the disease that may interfere with chemotherapy evaluation.

Chemotherapy regimens. The combined group and venous group were given paclitaxel, cisplatin and 5-fluorouracil (TCF), modified TCF (paclitaxel, oxaliplatin and 5-fluorouracil/tegafur), folinic acid, 5-fluorouracil and oxaliplatin (FOLFOX) or modified FOLFOX (folinic acid, tegafur and oxaliplatin). A total of 30 patients in the combined group received TCF or modified TCF regimens, and 32 patients received FOLFOX or modified FOLFOX regimens. In total, 32 and 34 patients, respectively, received these treatments in the venous group. Drug dosages were increased or decreased appropriately according to age, physical status and side effects. Chemotherapy was given every 28 days, and all patients received at least 2-3 cycles of chemotherapy.

Interventional approaches. The 5F-Yashiro vascular sheath was inserted following percutaneous femoral artery puncture using the Seldinger technique (9), then hooked onto the celiac axis and linked to an external high-pressure injector to perform high-pressure angiography. If the target vessel diameter was smaller than the 5F-Yashiro catheter or was too difficult to super-select for the 5F-Yashiro catheter, a micro godet was used to achieve intubation. The catheter was inserted into the left gastric artery, left under the phrenic artery and splenic artery for cancer of the cardiac and fundus, and through the left and right gastric artery for cancer of the gastric fundus and lesser curvature. For tumors located in the greater curvature, angiography of the gastroduodenal artery, right gastroepiploic artery and splenic artery was performed. Angiography of the gastroduodenal artery and right gastric artery was performed for gastric antrum cancer. Arteries which supplied blood to the tumor were selected, and the target artery was identified for chemotherapy and embolism according to the results of angiography. When liver metastases were present, chemoembolization of the hepatic arteries was performed. Following embolization, angiography was performed to ensure there were no feeding arteries (Fig. 1). In the present study, each patient underwent at least 1 interventional treatment and the average number of 
Table I. Clinicopathological characteristics of the two groups.

\begin{tabular}{|c|c|c|c|}
\hline Variable & Combined group $(n=62)$ & Venous group $(n=66)$ & P-value \\
\hline Age, years & $63.0 \pm 11.2$ & $60.4 \pm 12.5$ & $0.220^{\mathrm{a}}$ \\
\hline Sex, n (\%) & & & $0.387^{\mathrm{b}}$ \\
\hline Male & $50(80.6)$ & $49(74.2)$ & \\
\hline Female & $12(19.4)$ & $17(25.8)$ & \\
\hline Site of lesion, n (\%) & & & $0.288^{\mathrm{b}}$ \\
\hline Cardia & $44(71.0)$ & $38(57.6)$ & \\
\hline Gastric fundus & $9(14.5)$ & $14(21.2)$ & \\
\hline Gastric antrum & $9(14.5)$ & $14(21.2)$ & \\
\hline Degree of differentiation, $\mathrm{n}(\%)$ & & & $0.533^{\mathrm{b}}$ \\
\hline Poor & $22(35.5)$ & $20(30.3)$ & \\
\hline Moderate-well & $40(64.5)$ & $46(69.7)$ & \\
\hline Stage, n (\%) & & & $0.170^{\mathrm{b}}$ \\
\hline IIIC-stage & $26(41.9)$ & $20(30.3)$ & \\
\hline IV-stage & $36(58.1)$ & $46(69.7)$ & \\
\hline Lymph node metastasis, n (\%) & & & $0.388^{\mathrm{b}}$ \\
\hline None & $7(11.3)$ & $4(6.1)$ & \\
\hline Local lymph node metastasis & $32(51.6)$ & $41(62.1)$ & \\
\hline Distant lymph node metastasis & $23(37.1)$ & $21(31.8)$ & \\
\hline Organ metastasis, n (\%) & & & $0.132^{\mathrm{b}}$ \\
\hline None & $41(66.1)$ & $35(53.0)$ & \\
\hline Yes & $21(33.9)$ & $31(47.0)$ & \\
\hline Chemotherapy regimen & & & $0.991^{\mathrm{b}}$ \\
\hline $\mathrm{TCF}^{\mathrm{c}}$ & $30(48.4)$ & $32(48.5)$ & \\
\hline FOLFOX $^{\mathrm{d}}$ & $32(51.6)$ & $34(51.5)$ & \\
\hline
\end{tabular}

${ }^{a}$ As assessed using a two-tailed homoscedastic Student's t-test. ${ }^{b} \mathrm{P}-\mathrm{v}$ alues were based on $\chi^{2}$ tests and Fisher's exact tests. ${ }^{\mathrm{c}} \mathrm{Refers}$ to TCF and modified TCF. ${ }^{d}$ Refers to FOLFOX or modified FOLFOX. TCF, paclitaxel, cisplatin and 5- fluorouracil; FOLFOX, folinic acid, fluorouracil, and oxaliplatin.

Table II. Short-term curative effect of the two groups.

\begin{tabular}{lccc}
\hline Response rate $(\%)$ & Combined group $(\mathrm{n}=62)$ & Venous group $(\mathrm{n}=66)$ & $\chi^{2}$ \\
\hline $\mathrm{CR}, \mathrm{n}$ & $0(0)$ & $0(0)$ & $\mathrm{P}$-value \\
$\mathrm{PR}, \mathrm{n}$ & $22(35.5)$ & $13(19.7)$ & $35(53.0)$ \\
$\mathrm{SD}, \mathrm{n}$ & $30(48.4)$ & $18(27.3)$ & 4.010 \\
$\mathrm{PD}, \mathrm{n}$ & $10(16.1)$ & $13(19.7)$ & 0.045 \\
ORR, $\mathrm{n}$ & $22(35.5)$ & 13 & 4 \\
\hline
\end{tabular}

$\mathrm{CR}$, complete response; PR, partial remission; $\mathrm{SD}$, stable disease; $\mathrm{PD}$, progressive disease; ORR, objective response rate.

treatments was 2.7. The short-term effects of chemotherapy were evaluated in all patients.

Prognostic indicator. Objective response rate (ORR), overall survival (OS) and time to symptomatic progression (TTP) were used as prognostic indicators.

Imaging indicators. Examination consisted of chest and abdominal enhanced CT or enhanced MRI and, if necessary, positron emission tomography (PET)-CT or emission (E)CT were performed. Prior to treatment, tumor size, tumor number and maximum tumor diameter were recorded. Patients underwent these examinations every 2-3 cycles of chemotherapy until either mortality or termination of the present study (December 10th, 2015).

Laboratory indicators. Multiple laboratory indices were analyzed in the patient groups, including routine blood 
A

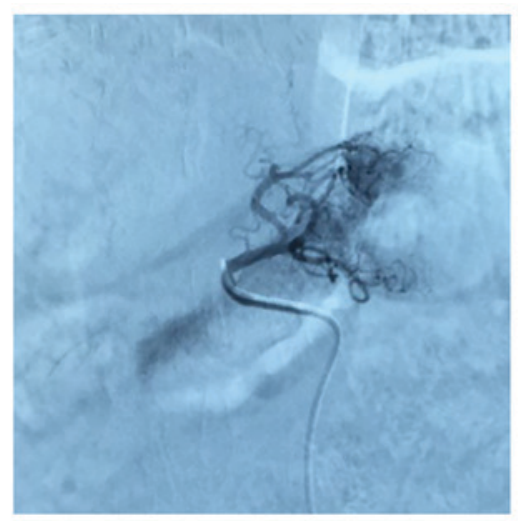

B

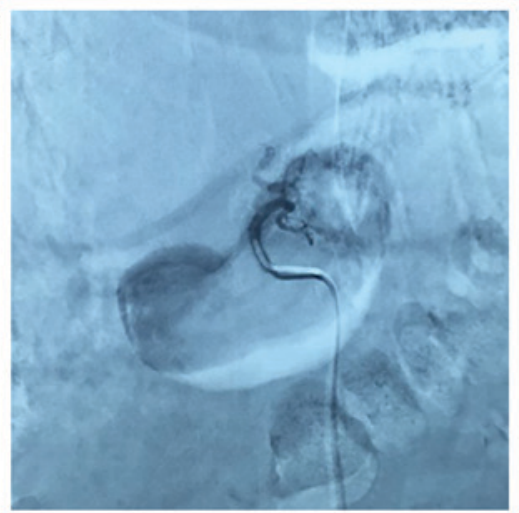

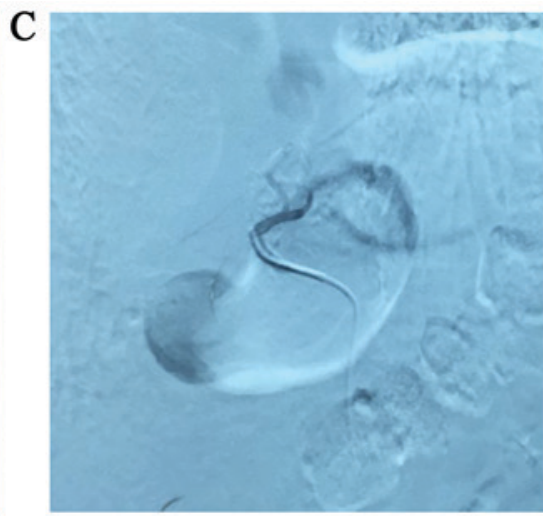

Figure 1. Regional intra-arterial chemoembolization process for gastric cancer. (A) Angiography of the left gastric artery reveals the cluttered tumor feeding arteries and distinct tumor staining. (B) Tumor staining visibly decreased following intra-arterial chemoembolization. (C) Tumor staining disappeared completely. Regional intra-arterial chemoembolization can effectively block tumor feeding arteries and remove the source of the blood supply to the tumor, thus the treatment has a more excellent antitumor effect. The images were screenshots of the original arteriogram.

samples, liver and kidney function, serum creatinine, blood urea nitrogen, and expression of carcino-embryonic antigen and carbohydrate antigen 19-9 (CA19-9). The neutrophil-to-lymphocyte ratio (NLR), platelet-to-lymphocyte ratio (PLR), changes in the NLR following 1 cycle of chemotherapy (cNLR) and changes in the PLR following 1 cycle of chemotherapy (cPLR) were also calculated.

The median NLR was 3.43 and the median PLR was 158.53 in the present study. The NLR and PLR were then divided into two groups, and the cNLR and cPLR into four groups, to investigate any influences on the OS of patients in the combined group. NLR was divided using a cutoff value of 3 into two groups ( $>3$ and $\leq 3$ ), and the PLR was divided using a cutoff value of 160 into two groups $(>160$ and $\leq 160$ ). The change in the cNLR was divided into four groups: Unchanged $\leq 3$; increased from $\leq 3$ to $>3$, decreased from $>3$ to $\leq 3$; and unchanged $>3$. Similarly, the change in the cPLR was divided into four groups: Unchanged $\leq 160$, increased from $\leq 160$ to $>160$, decreased from $>160$ to $\leq 160$, and unchanged $>160$.

Adverse reactions. According to the World Health Organization standard for side effects of a couplet anti-tumor treatment (10), the adverse side effects experienced by patients in the combined and venous groups were recorded and evaluated.

Treatment efficacy. In the present study, the curative effect was judged by the New Response Evaluation Criteria In Solid Tumors criteria (11), and consisted of a complete response (CR), partial remission (PR), stable disease (SD) and progressive disease (PD). Objective response rate (ORR) was calculated as: (CR+PR)/measurable number of cases) $\mathrm{x} 100 \%$.

Statistical analysis. Data were analyzed using SPSS version 21.0 (IBM Corp., Armonk, NY, USA). $\chi^{2}$ and Fisher's exact tests were used to assess qualitative data, and the unpaired Student's t-test was used to assess normally distributed quantitative data. The Kaplan-Meier method was used to calculate the survival time and the log-rank test was used to compare the different groups. The Kaplan-Meier method was also used for univariate analysis, and Cox regression analysis was employed for multivariate analysis. GraphPad Prism version 7 (GraphPad Software, Inc., La Jolla, CA, USA) was used to plot the survival curves. $\mathrm{P}<0.05$ was considered to indicate a statistically significant difference.

\section{Results}

Comparison of general clinical data. Age, sex, tumor site, pathological type, TNM stage, lymph node metastasis and organ metastasis status were not significantly different between patients in the two groups $(\mathrm{P}>0.05$; Table I).

Comparison of short-term curative effects. Of the 62 patients in the combined group, no patients were rated as having a CR, 22 as having a PR, 30 as SD and 10 as PD. The ORR was $35.5 \%$ (22/62). For the 66 patients in the venous group, these figures were $0,13,35$ and 18, respectively. The ORR was $19.7 \%$ (13/66), which was significantly different compared with the combined group $(\mathrm{P}=0.045$; Table II $)$.

Comparison of long-term curative effects. The survival time of the 128 patients was calculated and the total median (m)OS was 13.5 months [95\% confidence intervals $(\mathrm{CI})=12.42-14.58$ ] The mOS was $14(95 \% \mathrm{CI}=12.19-15.81)$ and 13 months (95\% CI=10.71-15.29) in the combined group and the venous group, and the 1-year and 2-year survival rates in the two groups were 45.2 and $9.7 \%$, and 40.9 and $6.1 \%$, respectively. There were significant differences between the survival curves of the combined group and the venous group ( $\mathrm{P}=0.044$; Fig. 2 ).

The total median (m)TTP was 9 months (95\% CI=7.60-10.40), and the mTTP was 10 months (95\% CI=8.38-11.62) and 6 months (95\% CI=3.60-8.40) in the combined group and venous group, respectively. TTP was significantly different between the two groups ( $\mathrm{P}=0.003$; Fig. 3).

Univariate analysis of $O S$ in the combined group. The purpose of the present study was to evaluate the efficacy and safety of combined intra-arterial and intravenous chemotherapy in the treatment of unresectable, advanced gastric cancer, and 
Table III. Univariate analysis of prognostic factors in the combined group.

\begin{tabular}{lccccc}
\hline Variable & & & Variable & mOS (months) & P-value \\
\cline { 1 - 1 } Sex & mOS (months) & P-value & & cPLR & 0.092 \\
Male & 14.5 & 0.794 & & Unchanged $>160$ & 12.5 \\
Female & 13.0 & & & Decreased from $>160$ to $\leq 160$ & 26.0 \\
Age, years & & & & Unchanged $\leq 160$ & 14.5 \\
$>60$ & 14.0 & 0.840 & & Increased from $\leq 160$ to $>160$ & 14.0
\end{tabular}

Table III. Continued.

Organ metastasis

Yes

No

Lymph node metastasis

None

Local lymph node metastasis

Distant lymph node metastasis

TNM stage

IIIC

IV

Differentiation

Poor

Moderate-well

Interventions

1-2

$\geq 3$

Chemotherapy regimen

TCF

FOLFOX

Site of lesion

Cardia

Gastric fundus

Gastric antrum

Tumor staining

Distinct

Not distinct

CEA, ng/ml

$>5$

$\leq 5$

CA19-9, U/ml

$>37$

$\leq 37$

NLR

$>3$

$\leq 3$

cNLR

Unchanged $>3$

Decreased from $>3$ to $\leq 3$

Unchanged $\leq 3$

Increased from $\leq 3$ to $>3$

PLR

$$
>160
$$

$\leq 160$

16.0

0.651

16.5

13.5

14.0

21.5

0.981

0.186

12.0

15.0

23.0

14.0

21.5

15.0

13.5

16.0

12.5

15.0

14.5

TNM, tumor-node-metastasis; mOS, median overall survival; TCF, paclitaxel, cisplatin and 5-fluorouracil; FOLFOX, folinic acid, fluorouracil, and oxaliplatin; CEA, carcino-embryonic antigen; CA19-9, carbohydrate antigen; NLR, neutrophil-to-lymphocyte ratio; PLR, platelet-to-lymphocyte ratio; cNLR, changes in the NLR following 1 cycle of chemotherapy; cPLR, changes in the PLR following 1 cycle of chemotherapy.

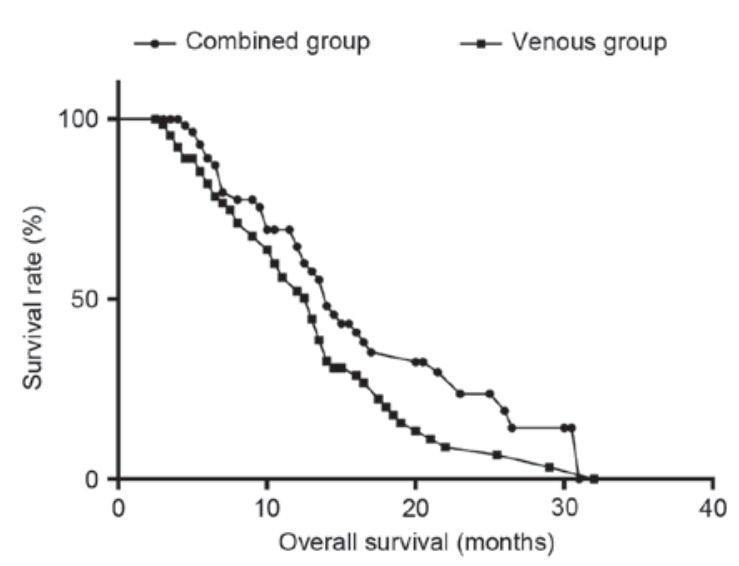

0.614

Figure 2. Overall survival of patients in the combined group and venous group. The median overall survival time was 14.0 months in the combined group and 13.0 months in the venous group, respectively $(\mathrm{P}=0.044)$.

0.010

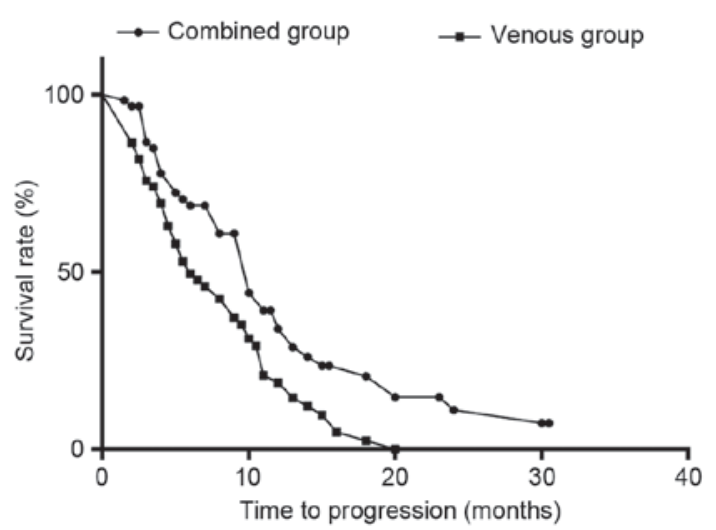

0.021

10.0

14.0

23.0

0.428

assess which patients are suitable for the combined treatment. Therefore, univariate analysis and multivariate analysis were conducted only for the combined group and only patients from the combined group were represented in the figures. 


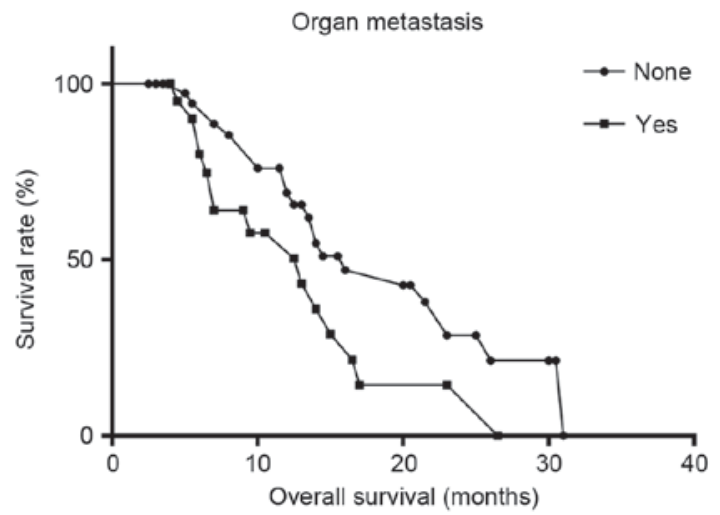

Figure 4. Overall survival of patients in the combined group with or without organ metastasis. The median overall survival time of patients with or without organ metastasis was 13.0 and 16.0 months, respectively $(\mathrm{P}=0.046)$.

CA19-9

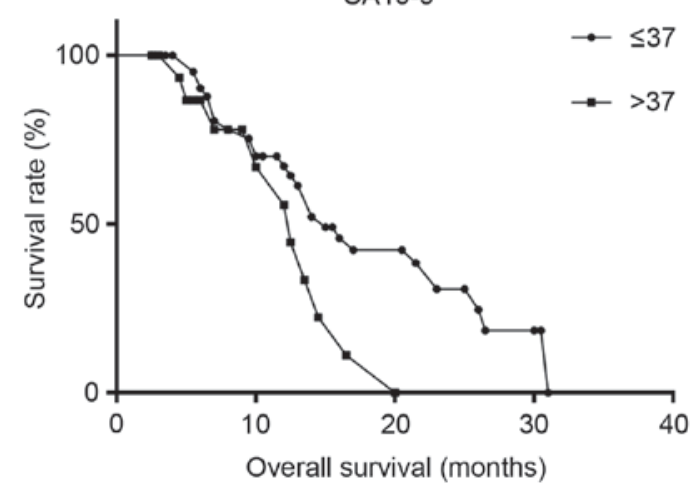

Figure 5. Overall survival of patients in the combined group with different CA19-9 levels. The median overall survival time of patients with CA19-9 $\leq 37 \mathrm{U} / \mathrm{ml}$ and CA19-9 $>37 \mathrm{U} / \mathrm{ml}$ was 15.0 and 12.5 months, respectively $(\mathrm{P}=0.036)$. CA19-9, carbohydrate antigen.

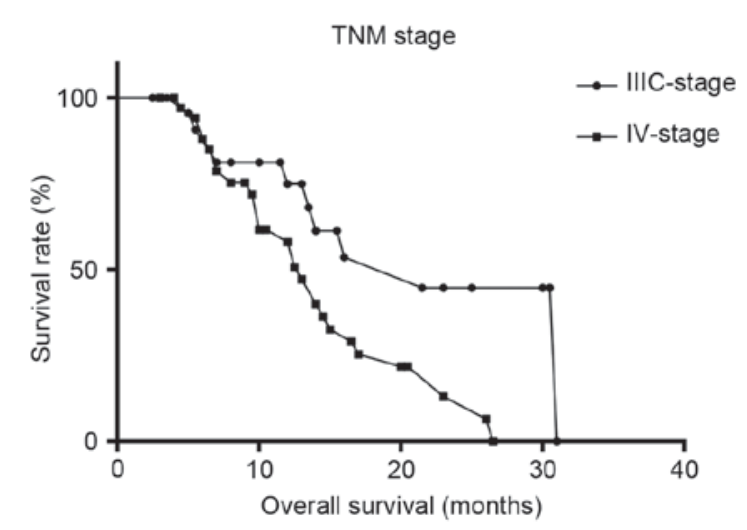

Figure 6. Overall survival of patients in the combined group with different tumor-node-metastasis stages. The median overall survival time of patients with III-C stage and IV-srage cancer was 21.5 and 13.0 months, respectively $(\mathrm{P}=0.017)$.

Univariate analysis revealed that organ metastasis $(\mathrm{P}=0.046)$, TNM stage ( $\mathrm{P}=0.017)$, CA19-9 levels $(\mathrm{P}=0.036), \mathrm{NLR}(\mathrm{P}=0.048)$, cNLR $(\mathrm{P}=0.021)$ and degree of tumor staining $(\mathrm{P}=0.010)$ were associated with patient OS in the combined group (Table III). In patients without organ metastasis, CA19-9 $\leq 37$ U/ml, TNM-IIIC

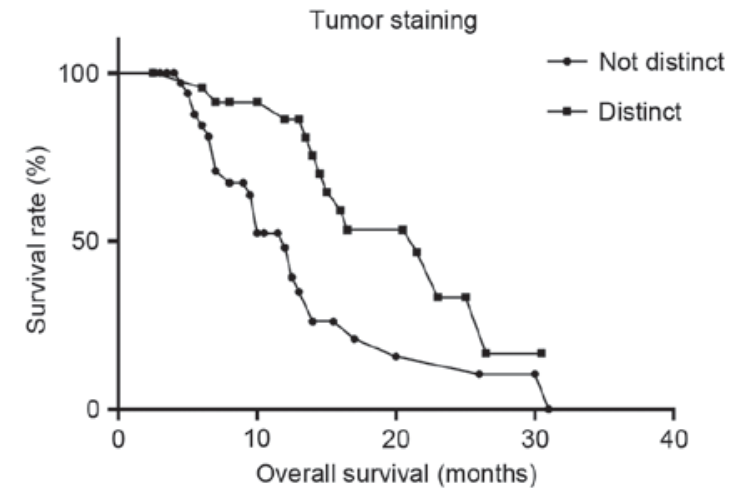

Figure 7. Overall survival of patients in the combined group with different degrees of tumor staining. The median overall survival time of patients with or without distinct tumor staining was 21.5 and 12.0 months, respectively $(\mathrm{P}=0.010)$.

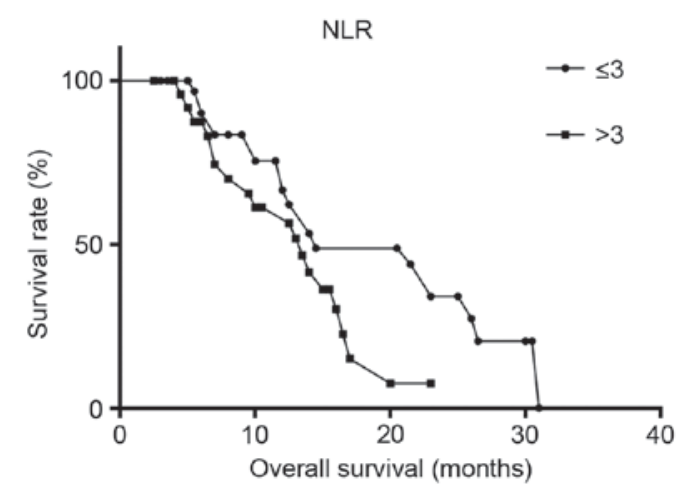

Figure 8. Overall survival of patients in the combined group with different NLR values. The median overall survival time of patients with NLR $\leq 3$ and NLR $>3$ was 14.5 months and 13.5 months, respectively $(\mathrm{P}=0.048)$. NLR, neutrophil-to-lymphocyte ratio.

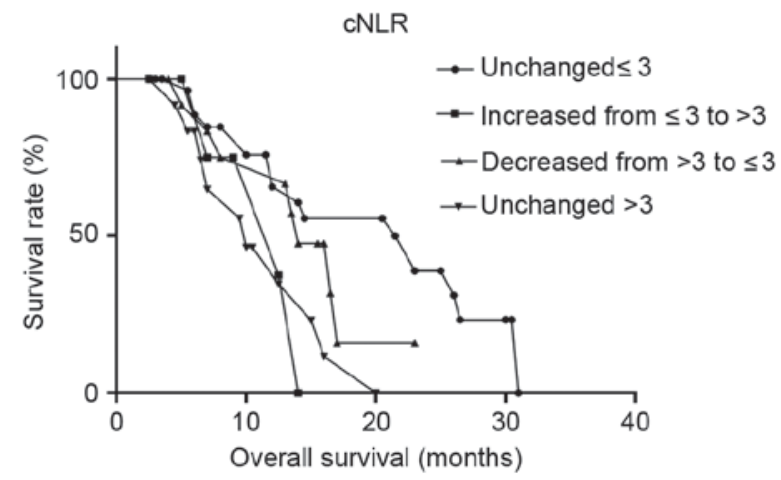

Figure 9. Overall survival of patients in the combined group with different cNLR values. The median overall survival time of patients with cNLR unchanged $>3$, decreased from $>3$ to $\leq 3$, unchanged $\leq 3$ and increased from $\leq 3$ to $>3$ was $10.0,14.0,23.0$ and 12.5 months, respectively $(\mathrm{P}=0.021)$. cNLR, changes in the neutrophil-to-lymphocyte ratio following 1 cycle of chemotherapy.

stage, NLR $\leq 3$, cNLR unchanged $\leq 3$ and distinct tumor staining, mOS was significantly increased (Figs. 4-9).

Multivariate analysis of $O S$ in the combined group. Multivariate analysis revealed that TNM-stage $(\mathrm{P}=0.025)$ 
Table IV. Multivariate analysis of prognostic factors in the combined group.

$95 \%$ CI for $\operatorname{Exp}(\beta)$

\begin{tabular}{lcccccc}
\cline { 5 - 7 } Variables & $\beta$ & $\mathrm{df}$ & P-value $^{\mathrm{a}}$ & $\operatorname{Exp}(\beta)$ & Lower & Upper \\
\hline TNM stage & -0.882 & 1 & 0.025 & 0.414 & 0.191 & 0.896 \\
Tumor staining & 0.869 & 1 & 0.015 & 2.383 & 1.184 & 4.798 \\
\hline
\end{tabular}

${ }^{a}$ Calculated using the Cox's forward logistic regression method. TNM, tumor-node-metastasis; CI, confidence interval.

Table V. Toxicity assessment for the two groups.

\begin{tabular}{|c|c|c|c|c|c|c|c|c|c|c|c|}
\hline \multirow[b]{2}{*}{ Adverse side effect } & \multicolumn{5}{|c|}{ Combined group $(\mathrm{n}=62)$} & \multicolumn{5}{|c|}{ Venous group $(n=66)$} & \multirow[b]{2}{*}{ P-value } \\
\hline & 0 & I & II & III & IV & 0 & $\mathrm{I}$ & II & III & IV & \\
\hline Leukopenia & 50 & 7 & 2 & 2 & 1 & 51 & 9 & 3 & 0 & 3 & 0.602 \\
\hline Thrombocytopenia & 58 & 2 & 2 & 0 & 0 & 64 & 0 & 1 & 0 & 1 & 0.372 \\
\hline Anemia & 55 & 2 & 5 & 0 & 0 & 55 & 6 & 4 & 1 & 0 & 0.424 \\
\hline Nausea and vomiting & 5 & 45 & 10 & 2 & 0 & 3 & 46 & 15 & 1 & 1 & 0.647 \\
\hline Hepatic inadequacy & 59 & 2 & 1 & 0 & 0 & 63 & 1 & 2 & 0 & 0 & 0.849 \\
\hline Neurological toxicity & 61 & 1 & 0 & 0 & 0 & 63 & 3 & 0 & 0 & 0 & 0.620 \\
\hline
\end{tabular}

and the degree of tumor staining $(\mathrm{P}=0.015)$ were independent factors affecting patient OS in the combined group (Table IV).

Comparison of adverse reactions. A statistical analysis of adverse reactions including myelosuppression, liver dysfunction, gastrointestinal reactions and neurotoxicity was performed. Of these, bone-marrow suppression and digestive-tract reactions were the most frequent toxic reactions. No treatment termination or mortality occurred as a result of toxic reactions in either group. There was no significant difference in the number of toxic reactions between the two groups $(\mathrm{P}>0.05$; Table $\mathrm{V})$.

\section{Discussion}

The concept of interventional treatment for gastric cancer was introduced in the 1980s, and has achieved a positive clinical effect. Interventional methods have developed from single perfusion chemotherapy to chemoembolization, and in order to further improve the curative effect, researchers have attempted to combine intravenous and intra-arterial administration. Zhang et al (12) used an arteriovenous combination of 5-fluorouracil, leucovorin, etoposide, oxaliplatin and epirubicin in patients with unresectable advanced gastric cancer, and Nakajima et al (13) used neo-adjuvant chemotherapy for inoperable gastric cancer via local and general delivery routes. These studies demonstrated that combined treatment significantly improves the local and systemic effects of treatment in patients with advanced gastric cancer.

In the present study, the mOS in the combined group was 14 months, and the 1-year and 2-year survival rates were
45.2 and $9.7 \%$, respectively; shorter than those reported by Shi et al (14), where the median survival time was 18 months and the 1-year and 2-year survival rates were 76.5 and $33.1 \%$, respectively. Potential reasons for this are as follows: i) The present study is retrospective and the overall response rates were obtained from medical histories, thus, there may have been individual subjectivity and diversity; ii) Of the 62 patients with advanced gastric cancer who underwent regional arterial infusion chemotherapy combined with intravenous chemotherapy, $\sim 58.1 \%$ of patients were TNM-IV stage, and $33.9 \%$ of patients had organ metastases, thus the overall effect was limited; iii) Patients in the combined group had an average of 2.7 interventional treatments, which was fewer than that in previous studies, including the study by Shi et al (14), where the patients underwent 7 cycles of interventional treatment on average.

In the present study, the combined group had an advantage in terms of the short-term and long-term effects of treatment compared with the venous group. The main reasons are as follows: i) Regional arterial infusion chemotherapy in the combined group boosted the drug concentration and maximized the effect of chemotherapy. In a study by Zhu and $\mathrm{Pu}(15)$, the concentration of 5-fluorouracil in portal venous blood following intra-arterial administration was 4-40-fold higher than that following venous administration, and high concentrations of 5-fluorouracil were maintained for a significantly longer time period. Therefore, compared with systemic chemotherapy only, combined venous and arterial chemotherapy concentrates the drug in question and prolongs the duration of its effect on tumor cells, resulting in an improved anti-cancer effect. ii) Embolization therapy in the combined group removed the source of the blood supply to 
the tumor, and resulted in tumor cell ischemia and necrosis. iii) Intra-arterial chemotherapy induces tumor cell apoptosis; for example, Tao and Zou (16) demonstrated that preoperative regional artery chemotherapy in patients with gastric cancer results in an improved curative effect, due to the inhibition of cell proliferation and induction of apoptosis. iv) For patients with distant metastases, in particular patients with hepatic metastases, chemoembolization effectively controls the disease. v) For patients with gastrointestinal bleeding, embolization effectively controls and prevents further hemorrhage, and improves quality of life and survival time.

Multivariate analysis revealed that distinct tumor staining was an independent factor affecting patient OS in the combined group, which is in line with the results of Zou and Tao (17), who revealed that distinct tumor staining and tumor blood supply consistency were associated with the effect of interventional treatment.

Cancer-associated inflammatory reactions have gained increased attention, and NLR and PLR are important in the prognosis of advanced gastric cancer (18-20). The present study emphasized the predictive effect of NLR, PLR, cNLR and cPLR in patients with advanced gastric cancer who received combined intra-arterial and intravenous chemotherapy; and univariate analysis revealed that patients with NLR $\leq 3$ and cNLR unchanged $\leq 3$ had an increased survival time.

NLR comprehensively reflects inflammation and immune status in patients with cancer, and increased NLR induces the inflammatory reaction and reduces anti-cancer activity. This results in the promotion of tumor growth, leading to a poor prognosis (21). Previous studies have reported that gastric cancer patients, with an elevated NLR have a poor prognosis $(21,22)$, which is in accordance with the results of the present study. The mechanisms underlying the predictive function of NLR in the prognosis of patients with gastric cancer is unclear, but may involve the following: i) Increased neutrophil number and function; with evidence suggesting that neutrophils contain and secrete cytokines and enzymes which may stimulate angiogenesis, increase tumor adhesion, inhibit apoptosis and facilitate distant metastasis $(23,24)$. Therefore, an elevated neutrophil count may stimulate tumor growth and progression (25). ii) Decreased lymphocyte count and function: Lymphocytes serve a crucial function in the process of cellular adaptive immunity, which plays an essential role in immunosurveillance, recognition and destruction of cancer cells $(26,27)$. Lymphopenia may reduce the anticancer function and lead to a worse outcome for cancer patients (28). Morris et al (29) have reported that a high number of tumorinfiltrating lymphocytes was strongly associated with favorable outcomes in patients with colon cancer, effectively confirming the aforementioned point.

In conclusion, regional intra-arterial chemoembolization combined with systemic chemotherapy in patients with unresectable, advanced gastric cancer is safe and effective, and patients with an earlier TNM stage and distinct tumor staining may achieve an improved clinical benefit. Compared with intravenous chemotherapy, combined chemotherapy in patients with unresectable, advanced gastric cancer has a positive curative effect in the long- and short-term. NLR and cNLR have a predictive effect on prognosis and the curative effect of chemotherapy in patients with advanced gastric cancer, and this inexpensive and convenient means of forecasting should be confirmed by further research and widespread use in the clinic.

\section{Acknowledgements}

The present study was supported by the National Natural Science Foundation of China (grant no. 81072966/H2902).

\section{Competing interests}

The authors declare that they have no competing interests.

\section{References}

1. Hamilton JP and Meltzer SJ: A Review of the Genomics of Gastric Cancer. Clin Gastroenterol Hepatol 4: 416-425, 2006.

2. Crew KD and Neugut AI: Epidemiology of gastric cancer. World J Gastroenterol 12: 354-362, 2006.

3. Zhu XD, Li J: Gastric carcinoma in China: Current status and future perspectives (Review). Oncol Lett 1: 407-412, 2010.

4. Zhang CW, Zou SC, Shi D and Zhao DJ: Clinical significance of preoperative regional intra-arterial infusion chemotherapy for advanced gastric cancer. World J Gastroenterol 10: 3070-3072, 2004.

5. Li M, Zhang J, Wang D, Zhong B, Tucker S, Lu S, Cheng J, $\mathrm{Cao} \mathrm{C}, \mathrm{Xu} \mathrm{J}$ and Pan $\mathrm{H}$ : A phase II study of intra-arterial chemotherapy of 5-fluorouracil, cisplatin, and mitomycin $\mathrm{C}$ for advanced nonresectable gastric cancer. Anticancer Drugs 20: 941-945, 2009.

6. Shchepotin IB, Cborny V, Hanfelt J and Evans SR: Palliative superselective intra-arterial chemotherapy for advanced nonresectable gastric cancer. J Gastrointest Surg 3: 426-431, 1999.

7. Edge SB, Compton CC (eds): The American Joint Committee on Cancer: the 7th edition of the AJCC cancer staging manual and the future of TNM. Ann Surg Oncol 6: 1471-4, 2010.

8. Oken MM, Creech RH, Tormey DC, Horton J, Davis TE, McFadden ET and Carbone PP: Toxicity and response criteria of the Eastern Cooperative Oncology Group. Am J Clin Oncol 5: 649-655, 1982 .

9. Seldinger SI: Catheter replacement of the needle in percutaneous arteriography; a new technique. Acta Radiol 39: 368-376, 1953.

10. Miller AB, Hoogstraten B, Staguet M and Winkler A: Reporting results of cancer treatment. Cancer 47: 207-214, 1981.

11. Eisenhauer EA, Therasse P, Bogaerts J, Schwartz LH, Sargent D, Ford R, Dancey J, Arbuck S, Gwyther S, Mooney M, et al: New response evaluation criteria in solid tumours: Revised RECIST guideline (version 1.1). Cancer 45: 228-247, 2009.

12. Zhang C, Li G, Fan C, Xu J, Cao J, Liu S and Li N: Comparison of efficacy of different route of administration of chemotherapy on unresectable, advanced gastric cancer. World J Surg Oncol 10: $162,2012$.

13. Nakajima T, Ishihara S, Motohashi H, Kitamura Y, Nakajima Y, Fujii M, Tokunaga A, Matai K, Anzai $\mathrm{H}$ and Nishi M: Neo-Adjuvant chemotherapy for inoperable gastric cancer via local and general delivery routes (FLEP Therapy). In: Cancer Treatment An Update. Springer Paris (ed.) Germany, pp411-413, 1994.

14. Shi DH, Cao JM, Gao DZ, Xu J, Kong WD, Li CL and Wang ZQ: Modified FOLFOX regimen combined with interventional therapy for the treatment of advanced gastric cancer: A clinical study. Intervent Radiol 18: 759-762, 2009.

15. Zhu ZD and Pu YD: The study of pharmacokinetics of 5-Fu after left gastric artery intra-arterial infusion in treatment of gastric carcinoma. Chin J Bases Clin General Surg 8: 26-28, 2001.

16. Tao HQ and Zou SC: Effect of preoperative regional artery chemotherapy on proliferation and apoptosis of gastric carcinoma cells. World J Gastroenterol 8: 451-454, 2002.

17. Zou SC and Tao HQ: Several questions related on preoperative regional arterial chemotherapy for gastric and colorectal cancer. World Chin J Digest 15: 477-481, 2007.

18. Gu X, Gao XS, Cui M, Xie M, Peng C, Bai Y, Guo W, Han L, $\mathrm{Gu} \mathrm{X}$ and Xiong W: Clinicopathological and prognostic significance of platelet to lymphocyte ratio in patients with gastric cancer. Oncotarget 7: 49878-49887, 2016. 
19. Cho IR, Park JC, Park CH, Jo JH, Lee HJ, Kim S, Shim CN, Lee H, Shin SK, Lee SK and Lee YC: Pre-treatment neutrophil to lymphocyte ratio as a prognostic marker to predict chemotherapeutic response and survival outcomes in metastatic advanced gastric cancer. Gastric Cancer 17: 703-710, 2014.

20. Yamanaka T, Matsumoto S, Teramukai S, Ishiwata R, Nagai Y and Fukushima M: The baseline ratio of neutrophils to lymphocytes is associated with patient prognosis in advanced gastric cancer. Oncology 73: 215-220, 2007.

21. Lee S, Oh SY, Kim SH, Lee JH, Kim MC, Kim KH and Kim HJ: Prognostic significance of neutmphil lymphocyte ratio and platelet lymphocyte ratio in advanced gastric cancer patients treated with FOLFOX chemotherapy. BMC Cancer 13: 350, 2013

22. Wang SC, Chou JF, Strong VE, Brennan MF, Capanu M, and Coit DG: Pre-treatment neutrophil to lymphocyte ratio independently predicts disease specific survival in resectable GE junction and gastric adenocarcinoma. Ann Surg 263: 292-297, 2016.

23. Bald T, Quast T, Landsberg J, Rogava M, Glodde N, Lopez-Ramos D, Kohlmeyer J, Riesenberg S, van den Boorn-Konijnenberg D, Hömig-Hölzel C, et al: Ultraviolet-radiation-induced inflammation promotes angiotropism and metastasis in melanoma. Nature 507: 109-113, 2014.

24. Wada Y, Yoshida K, Tsutani Y, Shigematsu H, Oeda M, Sanada Y, Suzuki T, Mizuiri H, Hamai Y, Tanabe K, et al: Neutrophi elastase induces cell proliferation and migration by the release of TGF- $\alpha$, PDGF and VEGF in esophageal cell lines. Oncol Rep 17: 161-167, 2007.

25. Tian $\mathrm{N}$ and Zhang PT: Relationship between neutrophilia and cancer progress or metastasis. Chin Canc 19: 470-476, 2010.
26. Schreiber RD, Old LJ and Smyth MJ: Cancer immunoediting: integrating immunity's roles in cancer suppression and promotion. Science 331: 1565-1570, 2011

27. Smyth MJ, Dunn GP and Schreiber RD: Cancer immunosurveillance and immunoediting: The roles of immunity in suppressing tumor development and shaping tumor immunogenicity. Adv Immunol 90: 1-50, 2006.

28. Ray-Coquard I, Cropet C, Van Glabbeke M, Sebban C, Le Cesne A, Judson I, Tredan O, Verweij J, Biron P, Labidi I, et al: Lymphopenia as a Prognostic Factor for Overall Survival in Advanced Carcinomas, Sarcomas, and Lymphomas. Cancer Res 69: 5383-5391, 2009.

29. Morris M, Platell C and Iacopetta B: Tumor-infiltrating lymphocytes and perforation in colon cancer predict positive response to 5-fluorouracil chemotherapy. Clin Cancer Res 14: 1413-1417, 2008.

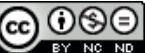

This work is licensed under a Creative Commons Attribution-NonCommercial-NoDerivatives 4.0 International (CC BY-NC-ND 4.0) License. 